\title{
Desarrollo de las úlceras en el pie diabético
}

\author{
Aurora Espinoza Valdez
}

Departamento de Ciencias Computacionales, CUCEI, Universidad de Guadalajara.

aurora.espinoza@cucei.udg.mx

\section{Beatríz Carely Luna Olivera}

Cómputo Científico y Matemáticas Aplicadas

de la Universidad de Papaloapan.

bcluna@unpa.edu.mx

Gualberto Solís Perales

Departamento de Electrónica, CUCEI, Universidad de Guadalajara. gualberto.solis@cucei.udg.mx

Resumen: El pie diabético constituye un problema de salud pública por su alta prevalencia y sus enormes costos. Debido a esto, es necesario investigar nuevos enfoques de prevención y tratamiento de las complicaciones vasculares del pie diabético, para entender el comportamiento del padecimiento y obtener información relevante. Por tal motivo, se requiere un acercamiento multidisciplinario que ayude a evitar complicaciones vasculares en las extremidades inferiores. En esta contribución describimos como el uso de herramientas matemáticas y en particular la teoría de grafos sirven para estudiar y describir el comportamiento del desarrollo de las úlceras en el pie diabético. Los resultados muestran que es posible describir la dinámica de las úlceras en el grafo incorporando información fisiológica de las úlceras.

Palabras clave: Pie diabético, grafo, úlcera. 


\section{Development of diabetic foot ulcers}

Abstract: The diabetic foot is a public health problem due to its high prevalence and enormous costs. Because of this, it is necessary to investigate new approaches of prevention and treatment of the vascular complications of diabetic foot, in order to understand the behavior of the disease and to obtain relevant information. Therefore, a multidisciplinary approach is required to help to prevent vascular complications in the lower extremities. In this contribution we describe how the use of mathematical tools and in particular graph theory serve to study and describe the behavior of the development of diabetic foot ulcers. The results show that it is possible to describe the dynamics of the ulcers in the graph incorporating physiological information of them.

Keywords: Diabetic foot, graph, ulcer.

\section{Introducción}

La diabetes mellitus es una enfermedad crónica que aparece cuando el páncreas no produce insulina suficiente o cuando el organismo no utiliza eficazmente la insulina que produce (Organización Mundial de la Salud, OMS). Actualmente, la diabetes afecta a más de 371 millones de personas en el mundo (OMS). Se considera que en nuestro país actualmente existen aproximadamente 10,603,220 diabéticos (Federación Mexicana de Diabetes, FMD) con una prevalencia comparativa de diabetes $15.59 \%$, y con 3,452,410 personas con diabetes no diagnosticadas. Los gastos médicos de una persona con diabetes son de dos a cinco veces más altos que los de una persona sin esta enfermedad. En los pacientes con diabetes el riesgo de muerte es al menos dos veces mayor que en las personas sin diabetes.

Uno de los efectos de la diabetes no controlada es la hiperglucemia (aumento de glucosa en la sangre), que con el tiempo daña gravemente muchos órganos y sistemas, especialmente los vasos sanguíneos (Kolluru 2012). Un 50\% de los 
pacientes mueren de enfermedad cardiovascular (OMS). Un problema frecuente en los pacientes diabéticos es el pie diabético, esto debido a las alteraciones vasculares observadas que muestran características especiales. El pie diabético, se define como infección, ulceración y destrucción de los tejidos profundos, asociadas con anormalidades neurológicas de diversa gravedad en las extremidades inferiores (OMS). La neuropatía de los pies (lesión microvascular de vasos sanguíneos que irrigan los nervios) combinada con la reducción del flujo sanguíneo incrementan el riesgo de úlceras de los pies, y en última instancia, amputación. La neuropatía diabética se debe a lesión de los nervios a consecuencia de la diabetes, y puede llegar a afectar a un $50 \%$ de los pacientes.

El control de la glucemia parece prevenir el desarrollo de microangiopatías (afectación de la función capilar) de la retina y el riñón, y probablemente retarda la evolución de neuropatías. Muchas lesiones del pie diabético se inician con síntomas de neuropatía. La neuropatía diabética incluye síntomas sensoriales menores, reducción de la velocidad de conducción nerviosa y resistencia a la falla de conducción isquémica, y la hipoxia nerviosa parece tener un papel significante en su origen.

De todas la amputaciones relacionadas con diabetes, $70-80 \%$ son precedidas por úlceras crónicas y hasta dos tercios experimentarán una segunda amputación (OMS). Se calcula que el tratamiento y atención básica de la diabetes permitirían prevenir hasta el $80 \%$ de las amputaciones de pies diabéticos (OMS). Esto es, las amputaciones de pie diabético se pueden disminuir mediante la modificación de factores como la neuropatía y el control glucémico, siendo este último el principal factor que causa un desorden en el proceso de angiogénesis para la generación de nuevos vasos sanguíneos (Lepäntalo et al. 2011). 


\section{Representación de las úlceras en un grafo}

El pie es particularmente vulnerable a daños circulatorios y neurólogicos, y el menor trauma puede causar úlceras o infecciones (Figura 1).

La Figura 2 indica las principales zonas del pie en las que aparecen con mayor frecuencia las úlceras. Las úlceras localizadas en la zona plantar tienen forma ovalada, son profundas, con bordes callosos y base granulada. Por lo contrario, las que aparecen en los dedos tienen bordes planos e irregulares y forma redondeada y es posible la afectación ósea. Existe un sistema de clasificación desarrollado por Wagner para la estadificación de las úlceras de pie diabético, que ha sido ampliamente aceptado, ver Figura 3 (Wagner 1979, Gordois et al. 2003):

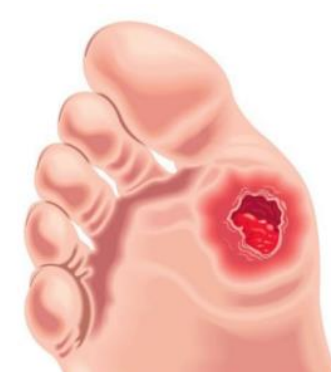

Figura 1. Úlcera neuropática en una posición típica bajo el metatarso y rodeada de callosidad. 


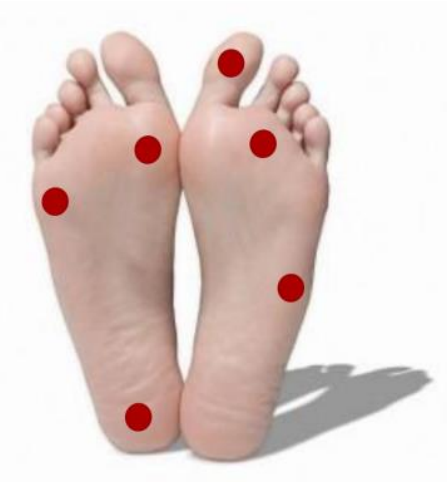

Figura 2. Puntos más susceptibles a la formación de úlceras en el pie diabético.

A fin de modelar un grafo para visualizar la dinámica de las úlceras en el cual se pueda incluir la información fisiológica, definimos un grafo.

Definición 1. Un grafo $G$ es una terna ordenada $(V(G), E(G), \psi G)$ que consiste de un conjunto no vacío $V(G)$ de vértices, de un conjunto $E(G)$ de aristas y de una función de incidencia $\psi_{G}$, que para cada arista se cumple:

- $\psi_{G}$ asocia la arista e, a un par de elementos de $V(G), \psi_{G}(e)=\{u, v\}$.

El grafo $G$ tiene vértices etiquetados con todas sus aristas orientadas, la orientación simboliza el cambio de estado de la úlcera. Cada vértice $v$ es etiquetado con la información fisiológica del estado de la úlcera, por la función $\mathrm{V}(\mathrm{G}) \rightarrow\left(\mathbf{R}^{+} \cup\{0\}\right)^{p}$, donde $p$ es el número de parámetros fisiológicos considerados para el desarrollo de úlceras. Representación de las úlceras en un grafo, ver Figura 4. 


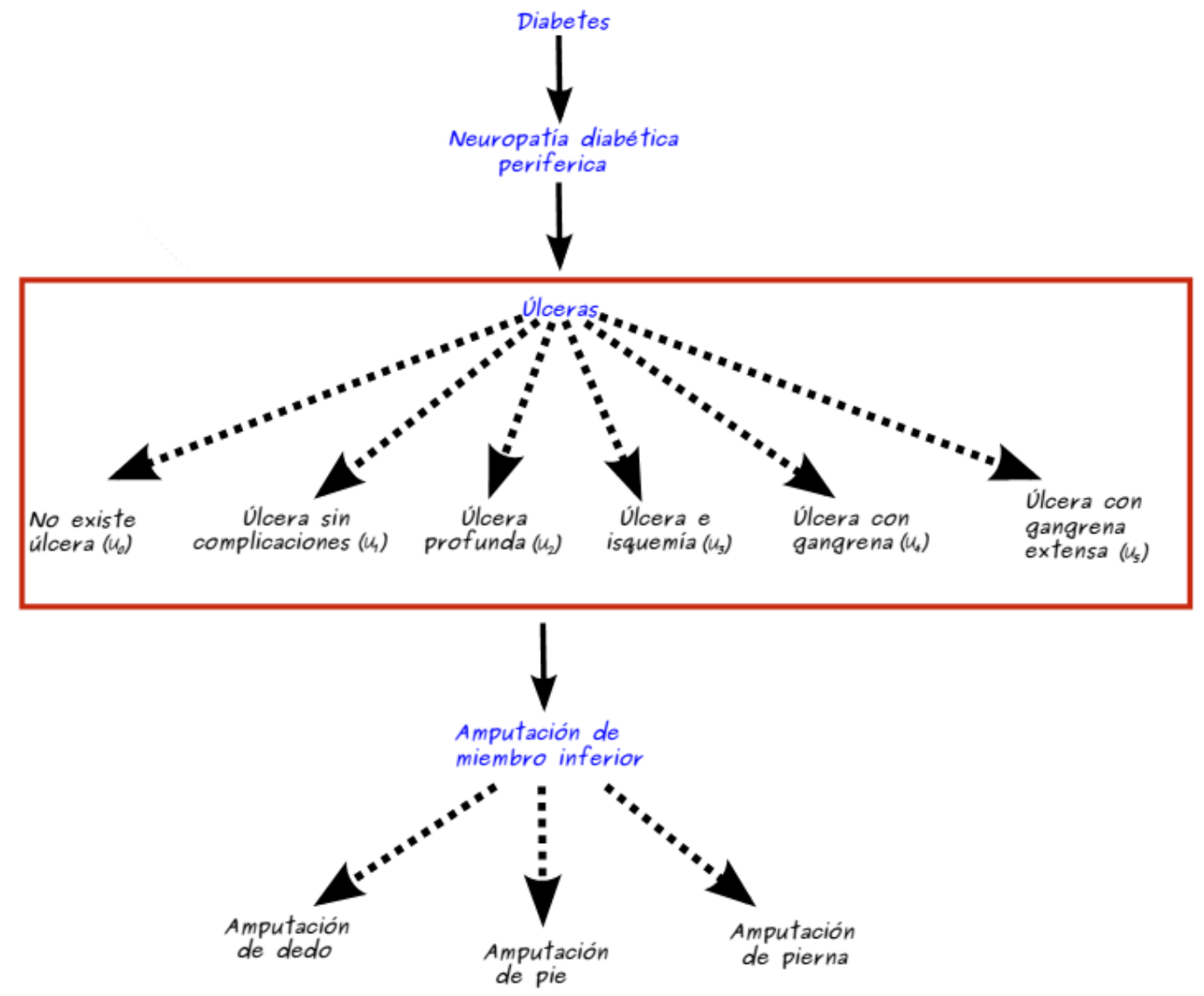

Figura 3. Clasificación de úlceras.

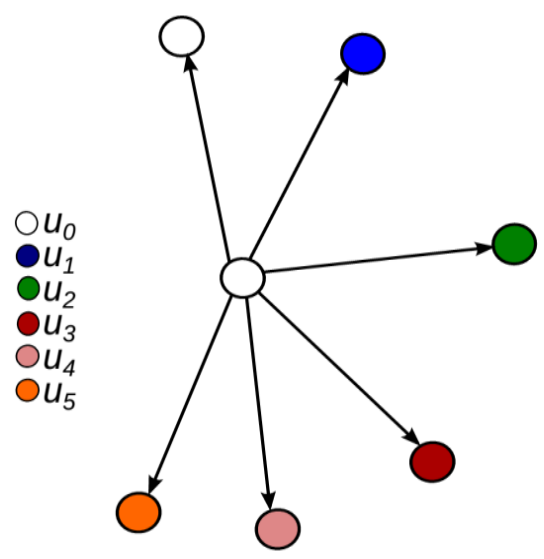

Figura 4. Grafo de esquema de las úlceras. 


\section{Resultados}

El cambio de estado de la úlcera depende de la concentración de glucosa $\mathrm{C}_{\text {gluc }}$ y el consumo de oxígeno $R_{\text {oxy }}$ en sangre (Buchwald 2011). Esto es, la concentración de oxígeno determina el estado de la úlcera (Lepäntalo et al 2011). Por otra parte, el tiempo en el que se puede dar el cambio de estado depende de varios factores que se tienen que considerar, pero no se cuenta con la suficiente información experimental al respecto para considerar el factor tiempo.

En base a datos experimentales de monitoreo durante 365 días en pacientes con úlceras, se determinaron los intervalos de concentración de oxígeno (Lepäntalo et al. 2011, Beckert et al. 2006):

$u_{0} \in(0.114,0.143) M m^{-3}, u_{1} \in(0.084,0.114) M m^{-3}, u_{2} \in(0.057,0.084) M^{-3}$, $u_{3} \in(0.043,0.057) M^{-3}, u_{4} \in(0.03,0.043) M^{-3}$ y $u_{5} \in(0.026,0.03) M^{-3}$.

En la Figura 5 se describe la dinámica de la evolución de las estados $u_{i}(i=$ $0,1,2,3,4,5)$ que se definen como sigue: Estado $u_{0}$, se puede cambiar a cualquiera de los 5 estados $u_{1}, u_{2}, u_{3}, u_{4}, u_{5}$; Estado $u_{1}$, se puede cambiar a los estados $u_{2}, u_{3}, u_{4}, u_{5}$ o bien regresar al estado $u_{0}$; Estado $u_{2}$, se puede cambiar al estado $u_{3}, u_{4}, u_{5} \circ$ bien regresar al estado $u_{0} \circ u_{1}$; Estado $u_{3}$ se puede cambiar al estado $u_{4}, u_{5} o$ bien regresar al estado $u_{1} O u_{2}$; Estado $u_{4}$, se puede cambiar al estado $u_{5}$ y no se regresa a ningún otro estado. 


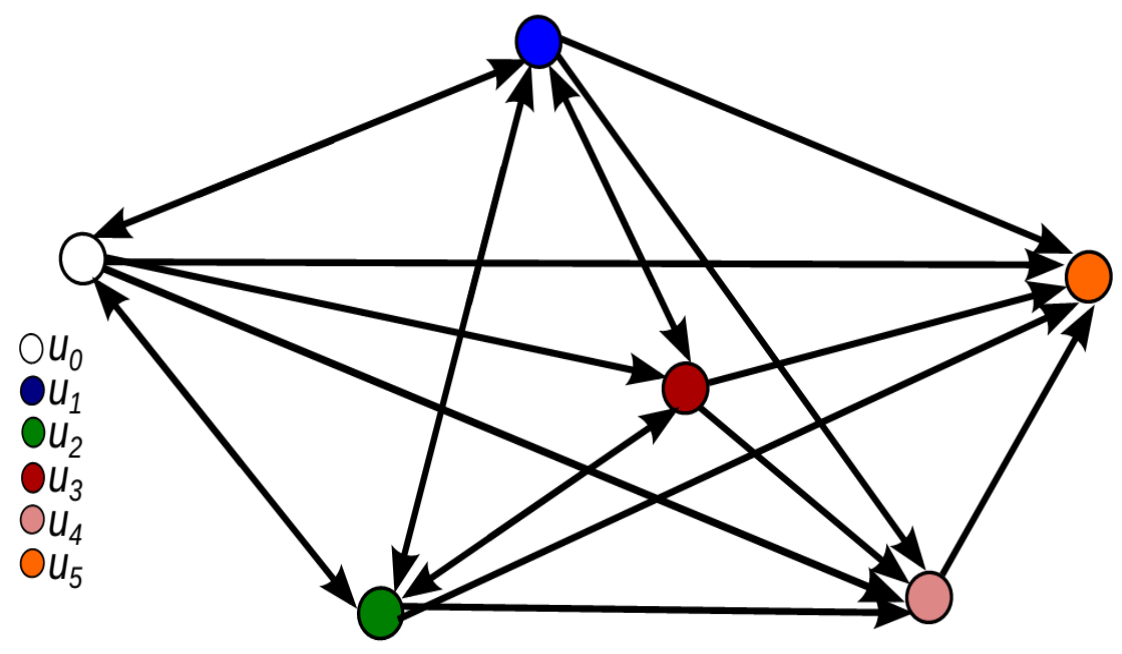

Figura 5. Dinámica de las úlceras.

El estado crítico de las úlceras es el $u_{3}$, ya que además de la úlcera existe isquemia y por lo tanto no se puede regresar a una piel sana, i.e. no es reversible hasta $u_{0}$. Existe un conjunto de posibles caminos en función de los parámetros fisiológicos, entonces de acuerdo a la dinámica que existe se determina el siguiente árbol que describe algunas de todas las posibles combinaciones 0 escenarios que puede tener un paciente con pie diabético (Figura 6). 


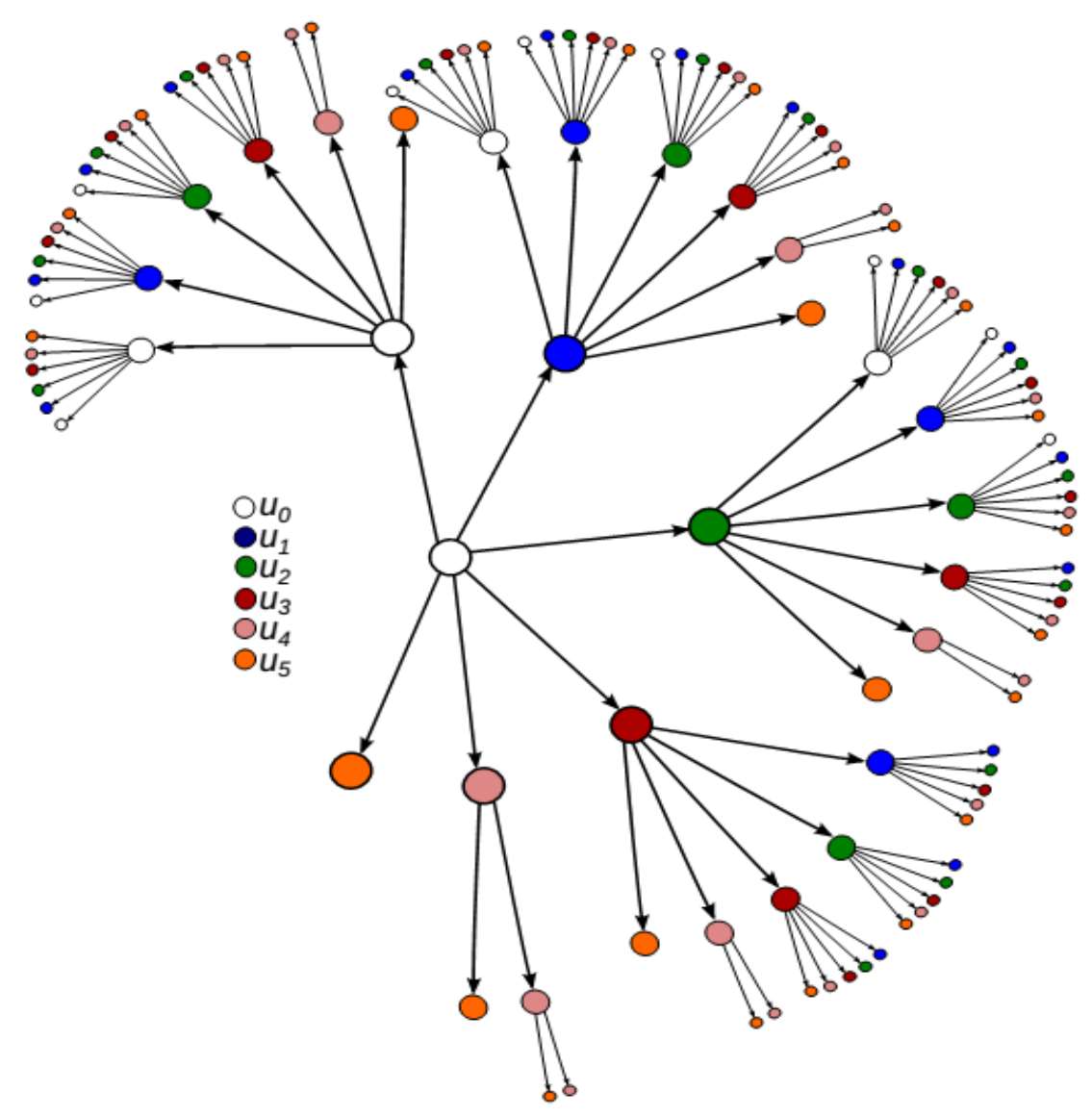

Figura 6. Posibles caminos.

\section{Conclusiones}

En este trabajo se muestra que el desarrollo de las úlceras en el pie diabético, se puede representar mediante un grafo $G$ con vértices etiquetados y aristas orientadas. El vértice incluyendo información fisiológica del principal parámetro de concentración de oxígeno. En base a esto, se analizó cada uno de los estados de las úlceras para determinar su reversibilidad, obteniendo que el estado crítico es el $u_{3}$ de las úlceras. En conclusión, la teoría de grafos permite modelar la dinámica de las úlceras, i. e., describe una red de como interactúan. 


\section{Referencias}

Beckert S., Witte M., Wicke C., Königsrainer A. and Coerper S. A new woundbased severity score for diabetic foot ulcers. A prospective analysis of 1,000 patients. Diabetes Care, Vol. 29 number 5, pp 988-992. 2006.

Buchwald P. A local glucose-and oxygen concentration-based insulin secretion model for pancreatic islets. Theoretical biology and medical modelling. Vol. 8. 2011.

Gordois A, Scuffham P, Schearer A, Oglesby A, Ash Tobian J. The health care cost of diabetic peripheral neuropathy in the US. Diabetes Care, Vol. 26, No. 6, pp. 1790-1795. 2003.

Kolluru GK, Bir SC, Kevil CG. Endothelial dysfunction and diabetes: efects on angiogenesos, vascular remodeling, and wound healimg. International Journal of vascular medicine. DOI:10.1155/2012/918267. 2012.

Lepäntalo M., Apelqvist J., Setacci C., Ricco J.B., de Donato G., Becker F., Robert-Ebadi H., Cao P., Eckstein H.H., De Rango P., Diehm N., Schimidli J., Teraa M., Moll F.L., Dick F., and Davies A.H. Chapter V: Diabetic Foot. European Journal of Vascular and Endovascular Surgery, Vol. 42(s2), pp s60- s74. 2011.

Tomake R.J. Editor. Assemble of the vasculature and its regulation. Berlin Birkhäuser. 2001.

Wagner FW Jr. A classification and treatment program for diabetic, neuropathic, and dysvascular foot problems. In Instruccional Course Lecturs. Sr. Luis, CV Mosby, American Academy of Orthopaedic Surgeons, pp 143-165. 1979. 


\section{Notas biográficas:}

Aurora Espinoza Valdez recibió el grado de Licenciada en Matemáticas Aplicadas en la Universidad Autónoma de Baja California (UABC), México, a finales de 2000. El grado de Maestra en Ciencias Aplicadas en la Universidad Autónoma de San Luis Potosí (UASLP), México, en 2005. El grado de Doctora en Ciencias en el Instituto Potosino de Investigación Científica y Tecnológica (IPICyT), México, a finales de 2010. Es miembro del Sistema Nacional de Investigadores (SNI), México. Desde el 02/2012 es Profesor Investigador del Depto. de Ciencias Computacionales en el Centro Universitario de Ciencias Exactas e Ingenierías de la Universidad de Guadalajara. Su interés de investigación es el modelamiento matemático de sistemas biológicos y sistemas dinámicos.

Beatríz Carely Luna Olivera estudió la Licenciatura en Matemáticas en la UDLA Puebla, realizó su maestría y doctorado en Ciencias con especialidad en Matemáticas Aplicadas en la Universidad Autónoma de San Luis Potosí. Actualmente trabaja en la Universidad del Papaloapan, en donde es profesora investigadora, es miembro del SNI, nivel I y tiene perfil PROMEP. Sus principales intereses son los estudios de sistemas dinámicos discretos, topología y dinámica de redes, y el análisis de redes que aparecen en diversos fenómenos químicos, médicos, biológicos, sociales, etc., así como también la divulgación de las matemáticas en todos los niveles educativos.

Gualberto Solís Perales es Ingeniero Electrónico egresado de la Facultad de Ciencias de la Universidad Autónoma de San Luis Potosí en 1995, Maestro y Doctor en Ingeniería Eléctrica en 1999 y 2002 respectivamente, ambos grados por la Facultad de Ingeniería de la Univesridad Autónoma de San Luis Potosí. Es miembro del Sistema Nacional de Investigadores desde el año 2002. Sus áreas de interés son el control no lineal de sistemas dinámicos, y particularmente en la sincronización de sistemas y redes de sistemas dinamicos y sus aplicaciones en sistemas biológicos, electrícos y electrónicos. Actualmente es Profesor Investigador en el Centro Universitario de Ciencias Exactas e Ingenierías de la Universidad de Guadalajara. 


\section{(c) (1) (2) (2)}

Esta obra está bajo una licencia de Creative Commons

Reconocimiento-NoComercial-Compartirlgual 2.5 México 